\title{
Emerging technologies for the detection of melanoma: achieving better outcomes
}

This article was published in the following Dove Press journal:

Clinical, Cosmetic and Investigational Dermatology

9 November 2012

Number of times this article has been viewed

\section{Cila Herman}

Department of Mechanical

Engineering, Johns Hopkins University, Baltimore, MD, USA
Correspondence: Cila Herman Department of Mechanical Engineering, Johns Hopkins University, 102 Latrobe Hall, 3400 North Charles Street, Baltimore, MD 21218-2682, USA

Tel + I 4I05164467

$\mathrm{Fax}+\mathrm{I} 4105167254$

Email cherman@jhu.edu
Abstract: Every year around 2.5-3 million skin lesions are biopsied in the US, and a fraction of these - between 50,000 and 100,000 - are diagnosed as melanoma. Diagnostic instruments that allow early detection of melanoma are the key to improving survival rates and reducing the number of unnecessary biopsies, the associated morbidity, and the costs of care. Advances in technology over the past 2 decades have enabled the development of new, sophisticated test methods, which are currently undergoing laboratory and small-scale clinical testing. This review highlights and compares some of the emerging technologies that hold the promise of melanoma diagnosis at an early stage of the disease. The needs for detection at different levels (patient, primary care, specialized care) are discussed, and three broad classes of instruments are identified that are capable of satisfying these needs. Technical and clinical requirements on the diagnostic instruments are introduced to aid the comparison and evaluation of new technologies. White- and polarized-light imaging, spatial and spectroscopic multispectral methods, quantitative thermographic imaging, confocal microscopy, Optical Coherence Tomography (OCT), and Terahertz (THZ) imaging methods are highlighted in light of the criteria identified in the review. Based on the properties, possibilities, and limitations of individual methods, those best suited for a particular setting are identified. Challenges faced in development and wide-scale application of novel technologies are addressed.

Keywords: Infrared imaging, thermography, melanoma detection and diagnosis, quantitative imaging, in vivo diagnostics

\section{Introduction and background}

Melanoma incidence is increasing at one of the fastest rates for all cancers in the US, with a current lifetime risk of one in $55 .{ }^{1}$ Every year, approximately 68,000 melanomas will be diagnosed, with around 8700 resulting in death. If melanoma is detected at an early stage, before the tumor has penetrated the epidermis, the 5-year survival rate is about $99 \%{ }^{2}$ However, the 5-year survival rate drops dramatically - to $15 \%$ - for patients with advanced disease. ${ }^{2,3}$ At present, there are no systemic treatments available that significantly extend the life span of patients with advanced melanoma; ${ }^{4,5}$ therefore, the key to extended survival is early detection and treatment. ${ }^{6,7}$ In order to enable early detection and diagnosis, avoid unnecessary biopsies, and ultimately reduce the cost of care, it is essential to develop accurate, sensitive, and objective quantitative diagnostic instruments that have the potential to have a deep impact on the disease. Dramatic advances in solid-state electronics and instrumentation, imaging hardware, computers, and software tools, including image-analysis techniques, have opened new avenues for quantitative imaging for the detection of melanoma. This review highlights and 
compares some of the emerging technologies that hold the promise of melanoma diagnosis. The emphasis of the review is placed on the technology aspect of the instrumentation, recent advances, challenges, and research needs.

While most cancers develop deeper in the body and cannot be viewed with the naked eye, melanoma most often develops on the skin and is easily visible. The challenge that remains is recognizing it early, so that treatment can be initiated before the tumor reaches a thickness of $0.76 \mathrm{~mm} .{ }^{8}$ Detecting melanoma with the human eye can be challenging, because of the diversity of possible presentations during the early stage of the disease. While trained dermatologists can reliably recognize advanced melanoma by visual inspection (relying on the Asymmetry, Border irregularity, Color variegation, Diameter $>6 \mathrm{~mm}$, Evolution [ABCDE] criteria) melanomas at their very early and most curable stages often mimic histologically benign look-alike lesions. Instruments that can capture and quantify information not readily available to the human eye and aid in discerning whether a lesion is benign or malignant could dramatically improve survival rates and reduce health-care costs. Early detection of melanoma relies on periodic (yearly) complete and thorough examinations by physicians, supplemented by more frequent (monthly) selfexams by patients. Both physicians and patients would benefit from accurate and reliable instruments able to yield objective and quantitative assessment of lesions. These scanners can serve as preliminary screening tools, allowing more time for the clinician and specialist to focus on lesions that are more likely to be malignant.

\section{Types of melanoma detectors based on end user}

Therefore, the long-term goals of research efforts when developing instruments for the noninvasive in vivo diagnosis of melanoma are to develop three key types of devices (scanners):

- Type I: Relatively low-cost, easy-to-use, handheld scanners for screening purposes (for self-exams of at-risk patients or screening in primary care facilities), providing an indication whether a lesion has a potential for malignancy (in this case, the patient would be advised to seek medical care with advanced diagnostics methods). Currently, full-body photography is the tool available for patients' self-exams, and this method relies on the subjective visual comparison of the real-life lesion and the previously photographed lesion. Modern technologies offer a range of more objective methods to aid the self-exams of patients.

- Type II: Precision scanners that provide accurate and detailed visual and/or quantitative characterization of individual lesions for use in specialized clinics by trained professionals, to aid decisions regarding the need for biopsy or the extent of surgery. The focus of current research efforts is the development of these instruments, and several promising techniques have emerged over the past two decades. The transition from laboratory demonstrations and small-sample pilot trials to clinical practice remains a challenge.

- Type III: Full-body-imaging systems that would ideally map the entire body surface of the patient in three dimensions, with sufficient resolution to provide three-dimensional (3D) information similar to the currently used digital full-body photography. This imaging system would serve as a catalog of all lesions. White-light imaging could be combined and coupled with one of the more advanced and sensitive imaging systems with advanced diagnostic capabilities. These imaging systems are essential to provide objective reference baseline information when tracking the evolution of lesions; however, they also hold the promise of diagnostic capabilities.

With advances in telecommunication and information technologies, telemedicine, the technology of providing clinical health care at a distance is becoming a reality. All three types of scanners would support telemedicine applications by allowing the development of an image repository for a patient as well as the tracking of the patient's lesions over time and enable long-term follow-up care. Since melanoma is becoming a widespread health problem, telemedicine combined with quality information from scanners would be an invaluable tool in geographic regions where specialized care is not easily accessible. It would also help in eliminating distance barriers and improving access to medical services that would often not be consistently available.

\section{Requirements}

Ideally, a melanoma-detection instrument (scanner) should satisfy a range of requirements:

1. The scanner should provide an objective assessment of the lesion by measuring some physical properties of the tissue (by quantitatively comparing healthy and diseased tissue) and lesion, or by comparing the parameters of the diseased tissue with a previously established scale in order to eliminate the subjective component in the interpretation of the results.

2. The outcome of the scanning and data-analysis process should be delivered in quantitative form (for example, by characterizing the malignant potential of skin lesions with a simple, numeric, quantitative scale), followed by 
a conclusion/recommendation regarding the nature of the lesion.

3. In its basic form (type I and type III scanners used for screening), the screening scanner would not require a trained radiologist for interpretation of the images. Data and images from a type II device would be both quantitative and qualitative, and allow analysis of the characteristic features of a lesion in more detail. Specialized training would be necessary for the interpretation of data delivered by type II scanners.

4. The device and the measurement method should be sensitive enough to deliver a strong signal even for very early stage melanoma lesions.

5. The measurement method should be robust and insensitive to differences in ambient and measurement conditions and local and individual variations of skin properties.

6. Ideally, measurement should be relative, in that it compares the properties of the lesion and the healthy tissue of the same subject, rather than comparing the responses of different individuals. In this way, the influence of individual variations of skin-tissue properties can be greatly reduced or even eliminated.

7. The scanner should be easy to use without sophisticated and specialized training (except for some applications of type II scanners), so that it is suitable for self-exams of patients between appointments.

8. Ideally, the device would not require perfect contact with the skin or the use of an immersion fluid for imaging.

9. The measurement should be noninvasive, by either not requiring irradiation of the tissue or keeping irradiation low, to minimize affecting or causing damage to the skin. Some scanning methods require, for example, irradiation with light, sound, or electromagnetic (EM) radiation, while others measure the radiation naturally emitted by the skin/human body. Other interactions with the skin of the patient, eg, thermostimulation by cooling, should be moderate, in order to avoid or minimize discomfort during the scanning process.

10. Ideally, the same measurement method should be used for scanning of individual lesions (type I and type II devices), as in a full-body scanner (type III).

11. The exclusion criteria should not limit the use of the device significantly.

12. The scanner should be reliable in operation, small in size and be low-cost, sensitive in terms of measurement capabilities, and robust in use, with a strong support base for manufacturing, customer support, and repair.

13. The scanner(s) should be easy to integrate into telemedicine applications and systems.

14. The scanner(s) should have the ability to interface with the Internet in order to deliver information and images into the patient's record.

15. The scanner should have the ability to interface with the manufacturer's database and update the image library as new lesions are scanned. The interaction with the manufacturer's system should also allow seamless updates of the software as the database and software are updated (as opposed to purchasing upgrades every couple of years, for example). As the calibration database is continuously enhanced and upgraded, the improvements should be made available to the end user continuously and seamlessly.

16. The technology should be readily available commercially within a short time (a few years, depending on the length of the FDA approval process) and should be reliable and relatively low-cost.

The imaging systems currently under development as candidates for melanoma detection satisfy different sets of requirements from this list: no single technology can satisfy all requirements at the same time. The emphasis in this review is on technologies that offer the potential of in vivo measurements, since in vivo diagnostic tools are essential and very much in demand in many fields of medicine and in melanoma diagnosis in particular. In general, they can provide guidance for surgical interventions by detecting lesion margins or allow for replacement of general biopsies of suspicious tissues by more targeted biopsies (typically one out of 30-40 lesions biopsied is a melanoma). In this way, in vivo imaging methods would reduce unnecessary tissue excisions and the associated pathology costs, as well as biopsy-associated risks and morbidity. Other clinical applications of these imaging instruments include the monitoring of the effect of therapies to tailor drug or radiation treatments based on individual responses of the patients. While a considerable amount of effort has been devoted to the development of type II methods and devices, much less effort has been dedicated to type I and type III scanners. The existence of an accurate and sensitive screening tool for self-exams of patients (similar to pressure cuffs or blood glucose monitors) would have a dramatic impact on the early detection of melanoma. Some methods are well suited for in vitro imaging and tests, which is useful when there is a need for fast processing of large numbers of samples. 
By quantifying the imaging outcomes and screening cases that are not likely to be malignant, the pathologist is able to devote more attention to the samples that indicate possible malignancy.

\section{Classification of detectors based on detection method}

\section{Direct contact methods vs noncontact methods}

The methods suitable for melanoma diagnosis can be classified as those that require direct contact between the radiation source, skin, and receiving optics, and methods that do not require direct contact (remote methods). Direct-contact methods sometimes require immersion fluids between the skin and the optics to avoid losses caused by the mismatch of the optical properties of the media through which the signal propagates. The use of immersion fluids adds complexity to the measurement, and these methods are typically not suitable for full-body scanners. Noncontact or remote measurements can be affected by the involuntary movement of the subject or even breathing, and they will require motion-tracking algorithms to be included into the image-analysis and processing steps when high-accuracy measurements are needed. Another classification is based on the need to apply external forcing to the investigated system before or during a measurement.

\section{Active vs passive methods}

Active methods involve forcing (heating, cooling, application of pressure, application of a tracer, or a contrast medium, etc) and measure the response of the system to the applied forcing. Passive methods characterize the system as is, ie, they measure steady-state properties. Passive methods are easier to apply; however, active methods may be faster, yield a stronger signal, and be more sensitive and accurate. The diagnostic method may require exposing the subject to an external source of radiation (X-rays, ultrasound, light, and measuring the reflected, scattered, or transmitted portion of that radiation) or measurement of the radiation naturally emitted by the subject. The latter method is easier to apply and does not require a radiation source (making the instrument smaller and less complex); however, there is little flexibility in influencing the nature or magnitude of the measurement signal.

\section{Image-forming vs quantitative methods}

Diagnostic methods in medicine can be classified as imageforming (information characterizing the lesion is contained in an image, such as a digital photograph, ultrasound image, or X-ray image) and quantitative methods that deliver a quantitative signal (for example, in the form of electric current or potential difference). Visual inspection using the ABCDE criteria is essentially an image-forming method, with the visual information regarding the characteristics of light reflected from the skin being detected by the human eye and processed by the brain. The human brain is capable of processing large amounts of visual information quickly, and after suitable training comparing this information with the mental database of images. The diagnosis has a subjective component, and the experience of the person conducting the analysis plays a significant role. Describing a visual image with numbers (quantifying it) to allow rigorous quantitative comparison with other images is a very complex process. Fully emulating the visual image analysis and decision-making process of the human brain with a computer is not possible at this stage of technical development. However, modern computer-based image-processing tools can aid the quantification process by reducing the vast amount of information contained in visual images to a limited amount of quantitative data relevant for a particular diagnosis. Quantitative methods deliver data that are easily quantified or compared (such as peaks in a radiation spectrum); however, they lack the subjective visual detail that visual information can easily convey.

An essential component of a melanoma-detection system is a calibration database that is proprietary to the manufacturer of the instrument. This database evolves as more information (test data) becomes available during the development and use of the instrument. The calibration database is established by comparing the measurement signal to the gold standard - the pathology result - for the evaluated lesion. The accuracy of detection is expected to improve over time as new lesions are added to the library of lesions. The lesion-classification outcomes will vary from method to method, and it is essential to "teach" the computer how to discriminate lesions based on the data accumulated in the calibration database. The lesion-classification outcomes are established using biostatistics methods. An example of this kind of database is the one established for MelaFind based on clinical studies.

\section{Stages of instrument development}

Instrumentation for the early detection of melanoma is complex, both in terms of hardware and software as well as clinical testing, and the development of a new detection or imaging system is a multidisciplinary effort accomplished in several stages: 
1. The first step is the proof-of-concept study, involving the development and testing of a laboratory prototype instrument in a laboratory environment on an experimental phantom, in vitro or on animal models.

2. This step is followed by modifying the hardware for clinical studies and demonstrating the feasibility of the method in patient studies with a limited number of subjects. Initially, the image and data analysis are manual; after the initial learning phase, the objective is to develop automated analysis and classification criteria in the development steps to follow, which is a challenging task.

3. Successful feasibility testing on a limited number of subjects is followed by the development of equipment suitable for clinical testing on a larger number of patients to establish the calibration database. Automated imageprocessing algorithms and data-processing software are developed based on the data collected in the calibration database (image library). The creation and initial validation of the database, software, and the decisionmaking criteria is an iterative process requiring testing on a large number of patients and assessment of a large number of benign and malignant lesions.

4. Once both the software and the hardware have reached the necessary level of technological readiness (reaching the accuracy and sophistication required for clinical evaluation), the FDA approval process can begin. In the US, the first step in the approval is the clinical assessment of the instrument to gather data for premarket approval (PMA) report and documentation.

5. After receiving PMA, the market launch of the instrument follows. For example, it took around 10 years for MelaFind to mature from early proof-of-concept efforts to PMA.

There are numerous hurdles along the path of new medical device development, and the journey is difficult, costly and time-consuming. In spite of the many sophisticated laboratory imaging systems developed for melanoma imaging described in the literature, the automated quantitative detection remained an elusive goal, primarily because of the cost, effort, and time needed to develop a sophisticated, sensitive, and reliable device. The recent launch of the MelaFind scanner for the detection of melanoma is a step in the right direction. The development of screening instruments remains a challenge for the future, and it will require significant investment of resources, both public and private, to evaluate different technologies in order to develop instruments best suited for a particular application. It appears that justifying the need for investing in diagnostic tools for skin cancer, which is directly visible on the skin surface, is more difficult than for other conditions that cannot be directly visualized by the naked eye.

\section{Electromagnetic radiation in medical diagnostics}

EM radiation is the most commonly used means of probing human tissue in medical diagnostic and imaging instruments (X-rays, Magnetic Resonance Imaging (MRI), digital photography, etc). EM radiation is a form of energy emitted or absorbed by matter, which behaves like a wave when traveling through space with the speed $c$. EM radiation is characterized by both electric and magnetic field components, which oscillate in phase perpendicular to each other and perpendicular to the direction of the propagation of the wave and energy. In vacuum, EM radiation propagates at the speed of light $-c_{0}=2.998 \times 10^{8} \mathrm{~m} / \mathrm{s}$. EM radiation has practically no inertia; therefore it can be used to analyze high-speed, unsteady processes in the human body. Its interaction with living tissue depends on the properties of radiation, the frequency $v$ (or wavelength $\lambda$ ), and the power. For radiation propagating in a particular medium, these two properties are related as $\lambda=\mathrm{c} / \nu$. EM radiation carries energy through space, away from the source towards the matter with which it interacts.

The EM spectrum is illustrated in Figure 1, in order of increasing frequency and decreasing wavelength. The blackbody radiation spectra for thermal radiation (left) and IR radiation (right) are depicted in the top portion of Figure 1. The longest wavelengths in the EM spectrum are called radio waves, followed by microwaves, IR radiation (responsible for thermal effects), visible light, ultraviolet radiation, X-rays, and gamma rays. The intermediate portion of the spectrum that extends from 0.1 to $100 \mu \mathrm{m}$ is called thermal radiation, and it includes portions of the ultraviolet and all of the visible and IR spectra, as shown in Figure 1. The human eye is sensitive to a relatively small window of wavelengths of EM radiation called the visible spectrum, which is in the range of $0.4-0.7 \mu \mathrm{m}$. The nature of the interaction of EM radiation with biological tissue depends on the properties of radiation: its power and frequency (wavelength). For longer wavelengths and lower frequencies (radio waves, microwaves, IR radiation), the interaction (and potential damage) with cells (and other materials) is characterized by heating effects associated with the radiation power. For shorter wavelength, higher-frequency radiation (ultraviolet frequencies and above), such as X-rays and gamma rays, the potential damage to the chemical structures and bonds and living cells by EM radiation can be far larger than that done by simple heating. 


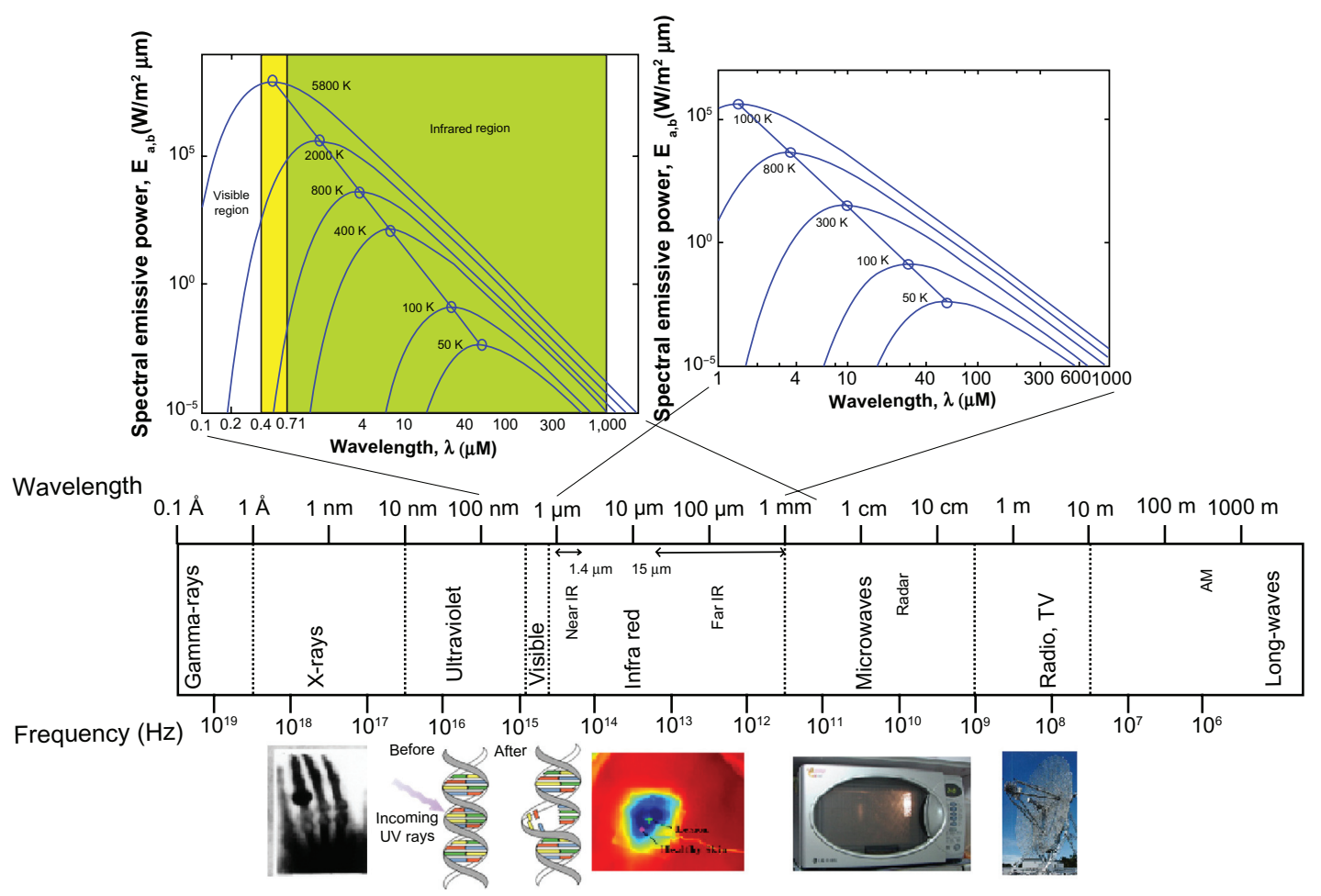

Figure I EM radiation spectrum shown in terms of wavelength and frequency, characteristic ranges used in medical diagnostics. Note: The black-body radiation spectrum for thermal and IR radiation is shown in the top portion of the image.

This can be attributed to the ability of photons to damage individual molecules chemically by affecting the chemical bonds. Whenever using EM radiation for probing human tissue in vivo, the frequency (wavelength) and power (and their combination) have to be selected in such a way to avoid affecting and damaging the tissue. More power can yield a stronger signal, but it is also more likely to cause damage; therefore, safety considerations will limit the magnitude of the probing radiation and thereby the measured signal.

A typical instrument or imaging system for medical diagnostic purposes consists of the source of radiation and a detector. The radiation emitted by the source (human body, sun, light bulb, laser source, etc) is reflected, absorbed, and scattered by human tissue, as illustrated in Figure 2A. Some forms of radiation, eg, $\mathrm{X}$-rays, can also be transmitted through the human body. The human body will also emit radiation in the IR domain of the spectrum (not visible to the naked eye), and the IR emission spectrum is shown in the top-right portion of Figure 1. The detector captures the reflected, scattered, transmitted, or emitted radiation. The reflection, transmission, scattering, and emission properties of healthy and diseased tissue will usually differ, and a sensitive detector will image or quantify this difference.

Radiation sources and detectors have evolved greatly over the past few decades, which has enabled measurements of very small signals, some that were too small to detect in the past. Therefore, the technology advances may allow measurements today that were impossible in the recent past. Depending on the wavelength range used for sensing, the design and complexity of the radiation source and the detector can vary greatly, and so do the challenges and costs involved with building the imaging hardware. For example, sources and detectors for X-ray radiation instruments in medical diagnostics are well established and readily available, whereas $\mathrm{THz}$ sources and detectors suitable for medical applications
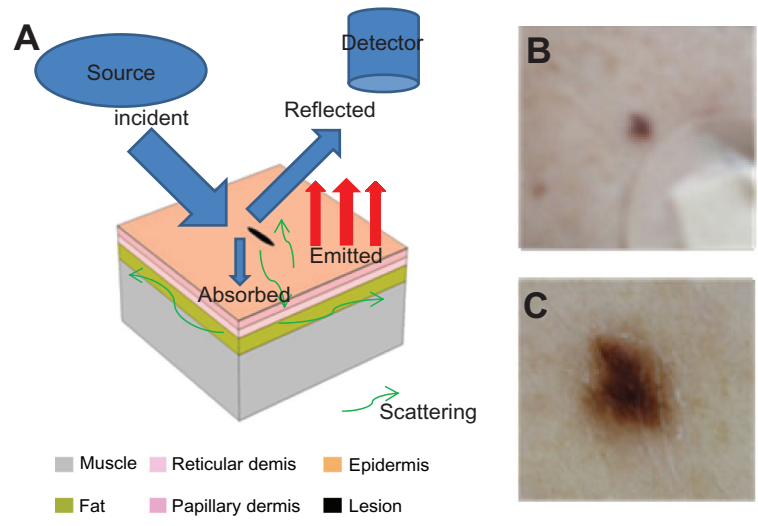

Figure 2 (A) Interaction of radiation with tissue (absorption, reflection), radiation source, and detector; (B) digital white light, and (C) dermoscopy images of a melanoma lesion, capturing the reflected portion of the incident EM radiation. 
emerged on the market relatively recently. Older technologies are often less costly, readily available, and already have good distribution and support (repair) networks. This is especially the case when a technology is widely used in other fields, such as IR imaging, with an established use in night-vision systems. Reliability testing and cost may initially pose a challenge when trying to rely on a new technology. An essential component of most medical instruments nowadays is a computer that stores, analyzes, and displays data to the user and interfaces the Internet for information access and storage. Over the past few decades, computer hardware and software have experienced dramatic advances, allowing fast processing of large amounts of complex data, which is essential for medical diagnostic systems. Through access to the Internet, safe, centralized storage and sharing (as needed) of large amounts of data have become a reality.

\section{Melanoma-detection methods}

The majority of cutaneous melanomas appear as pigmented lesions of the skin. The most effective screening tool for detection of atypical lesions and the standard of care, established 27 years ago, has been visual examination: assessing the lesion according to the $\mathrm{ABCDE}$ criteria, introduced in $1985 .{ }^{9,10}$ The last criterion, evolution, was added in 2004, after recognizing the importance of the changes in size and shape of the lesion in the screening process. ${ }^{9}$ The source of radiation is white light (from natural or artificial sources), and the radiation reflected by the skin is detected by the human eye. The diagnostic accuracy of the ABCDE criteria has been verified in clinical practice; however, it only provides qualitative guidelines for melanoma identification and yields high rates of false positives and frequent false negatives. Studies suggest that almost one out of three melanomas could not be diagnosed using these criteria. ${ }^{10}$ Over the past three decades, there have been improvements in imaging tools for melanoma detection; however, clinicians still primarily rely on their eyes, experience, and clinical judgment. Therefore, accurate, sensitive, objective, and quantitative instruments to aid the diagnosis of melanoma are still needed in clinical practice. In addition to this, screening tools that would alert patients during self-exams, coupled with the ability to store images and data online in a repository for long-term follow-up, would be invaluable in reducing the mortality associated with the disease. The melanoma-detection methods discussed in this review are summarized in Table 1 and their key features are indicated in the table.

\section{Digital photography and dermoscopy}

Current in vivo imaging tools commonly used by dermatologists are digital photography (total cutaneous imaging or the imaging of individual lesions) and dermoscopy. In digital photography, serial images recorded over time (photographic follow-up) are compared in order to find changes in size, shape, and color of pigmented lesions that might suggest malignancy. It was found that the relative change of a lesion is a sensitive marker for early melanoma., ${ }^{4,-10,14}$ However, additional studies also showed that only $35 \%$ of diagnosed melanomas were identified on the basis of the relative change. ${ }^{11,12}$

Table I Melanoma detection methods considered in this review and their key features

\begin{tabular}{|c|c|c|c|c|c|c|c|}
\hline Method & Type & Quantitative & $\begin{array}{l}\text { Image- } \\
\text { forming }\end{array}$ & $\begin{array}{l}\text { Activel } \\
\text { passive }\end{array}$ & $\begin{array}{l}\text { Contact/ } \\
\text { remote }\end{array}$ & $\begin{array}{l}\text { Radiation } \\
\text { type }\end{array}$ & $\begin{array}{l}\text { Technology } \\
\text { readiness }\end{array}$ \\
\hline $\begin{array}{l}\text { Digital } \\
\text { photography }\end{array}$ & I, II, III & No & Yes & Passive & Remote & $\begin{array}{l}\text { White } \\
\text { light }\end{array}$ & Available \\
\hline Dermoscopy & II & No & Yes & Passive & Both & $\begin{array}{l}\text { Polarized } \\
\text { light }\end{array}$ & Available \\
\hline MSS & I, II & Yes & Yes & Active & Contact & $\begin{array}{l}\text { Different } \\
\text { wavelengths }\end{array}$ & PMA \\
\hline MSF & I, II & Yes & $\begin{array}{l}\text { Usually } \\
\text { no }\end{array}$ & Active & Contact & $\begin{array}{l}\text { Different } \\
\text { wavelengths }\end{array}$ & $\begin{array}{l}\text { Research } \\
\text { phase }\end{array}$ \\
\hline $\begin{array}{l}\mathrm{THz} \\
\text { imaging }\end{array}$ & I?, II & Yes & Possible & Active & Both & $\mathrm{THz}$ range & $\begin{array}{l}\text { Research } \\
\text { phase }\end{array}$ \\
\hline $\begin{array}{l}\text { IR in spatial } \\
\text { domain }\end{array}$ & I, II, III & Yes & Yes & Can be both & Remote & $\begin{array}{l}\text { Naturally } \\
\text { emitted IR }\end{array}$ & $\begin{array}{l}\text { Research } \\
\text { phase }\end{array}$ \\
\hline CSLM & II & No & Yes & Active & Contact & $\begin{array}{l}\text { Different } \\
\text { wavelengths }\end{array}$ & Available \\
\hline OCT & II & No & Yes & Active & Contact & Near-IR & $\begin{array}{l}\text { Research } \\
\text { phase }\end{array}$ \\
\hline
\end{tabular}

Abbreviations: CSLM, confocal scanning laser microscopy; IR, infrared; MSF, multispectral imaging in the frequency domain; MSS, multispectral imaging in the spatial domain; OCT, optical coherence tomography; PMA, premarket approval. 
Dermoscopy involves the use of a handheld microscope and polarized light, which allows imaging of the deeper structures of the skin lesions. Reported sensitivity and specificity of dermoscopy are $98 \%$ and $68 \%$, respectively. ${ }^{12}$ Dermoscopy has been reported to improve the diagnostic accuracy for melanoma by $5 \%-30 \%$. However, studies have also confirmed that diagnostic accuracy is heavily dependent on the operator's experience, ${ }^{11-14}$ and both techniques are highly subjective, without broadly applicable standards or quantitative criteria. A digital photo and a dermoscopy image of a melanoma lesion are displayed in Figure 2B and C.

Whole-body photography (also referred to as full-body photography), which is essentially white-light, high-resolution digital photography, has become an important aid in patient self-exams. It involves the taking of photographs of the entire body, in order to identify suspicious lesions that may be malignant melanomas. This method is frequently used for high-risk patients. The patient uses an album with a set of professional, high-resolution photographs to compare the appearance of the lesion at the time of the self-exam with a baseline recorded earlier. Photographs of single lesions are used for the monitoring of a particular lesion when biopsy does not seem warranted. The expectation is that the use of photography combined with visual inspection will reduce unnecessary biopsies and allow for early detection of melanoma.

As far as insurance coverage is concerned, photographic methods (or other imaging techniques) in melanoma screening are often still considered to be experimental or investigational, since there is insufficient published clinical data to support a benefit for high-risk patients, which is coupled with the lack of standardization of methods and guidelines regarding when a lesion needs to be biopsied as opposed to monitored. Many insurance companies claim that there is currently a lack of sufficient evidence of improved patient outcomes (early detection or reduction in numbers of unnecessary biopsies) to justify the additional cost of imaging, and so many high-risk patients are forced to pay for the imaging (for example, total-body photography) out of pocket. However, considering the costs for treating advanced melanoma and the outcomes, it seems that encouraging the use of imaging in diagnostics would make sense and reduce morbidity and mortality as well as health-care costs on the long run.

Commercial systems for assessment of lesions using digital photography already exist; however, they are not in widespread use. Implementing the ABCDE diagnostic criteria in an automated evaluation algorithm on a computer is extremely challenging and has not yet been accomplished because of the diversity and complexity of lesions and images. Any commercial system to be used as a diagnostic tool in clinical practice would need extensive and expensive validation in clinical trials and FDA approval. With enhancements in imaging and computer technologies as well as image-processing software, the method of the future would be high-resolution 3D mapping of the entire body as a periodic screening tool. Ideally, the photographic imaging technique would be coupled with another more quantitative diagnostic technique.

\section{Multispectral imaging}

Spectral methods fall into the class of emerging new technologies that are currently being investigated to determine their ability to diagnose melanoma. Multispectral information can be acquired and analyzed both in the spatial domain (detector measures radiation intensity at a particular point or region) and the spectral domain (detector captures radiation intensity as function of wavelength - spectrum for the selected point or domain). The imaging equipment and the signal-analysis methods strongly depend on the approach taken. These methods hold the promise of offering quantitative criteria for melanoma diagnosis.

\section{Multispectral imaging in the spatial domain}

One of the most significant advances in the detection of melanoma over the past decade has been the development of the MelaFind ${ }^{15}$ technology by Mela Sciences. ${ }^{16}$ After a 2-year approval process, this technology received PMA (P090012) from the FDA for use in the US in 2011. The PMA approval was followed by approval for sale of the system in the European Union as well.

MelaFind delivers objective additional information (based on quantitative characterization of lesions) about clinically atypical cutaneous pigmented skin lesions with either clinical or historical characteristics of melanoma during skin examinations. ${ }^{17-21}$ The role of the instrument is to aid the decision of the dermatologist regarding diagnosis and the need for biopsy. The multicenter blinded study, using histologic data as a reference standard, supporting the PMA application for MelaFind involved 1383 patients in the US (enrolled from January 2007 to July 2008). The study demonstrated a sensitivity of over $98 \%$ of MelaFind (95\% lower confidence bound) with a biopsy ratio of 10.8:1. ${ }^{22}$ Most lesions were thin melanomas or borderline lesions. The MelaFind outcomes were binary: positive 
(lesion should be considered for biopsy) and negative (lesion should be considered for later evaluation). MelaFind had an average specificity of $9.5 \%$, whereas the specificity of the investigators was $3.7 \%{ }^{22}$ Clinicians participating in the study were blinded to the output of MelaFind, and patient care was managed based on clinical information. The lesion was considered positive when the prebiopsy dermatologic diagnosis was either melanoma or melanoma could not be ruled out. The study could not determine the true sensitivity of the participating dermatologists, since only lesions scheduled for biopsy were evaluated; melanomas missed by examining clinicians were not included in the study. The sensitivity of physicians can be assessed in longitudinal (long-term follow-up) studies, and such results were not available for this PMA because of the short time frame. Other studies list biopsy sensitivity values in the range of $86.7 \%$ $93.7 \% .^{22}$ In addition to this, a pilot study investigated the biopsy sensitivity of dermatologists in a reader study using 25 randomly selected melanomas and 25 non-melanomas. The readers were 39 dermatologists who did not participate in the clinical trial, and they reviewed the clinical history and the images of the lesions to decide whether to biopsy the lesion or rule out melanoma. The average biopsy sensitivity of the reader study was $78 \%,{ }^{22}$ a result similar to some prior studies. More details about the MelaFind study are available in the original publication. ${ }^{22}$

The MelaFind instrument characterizes and classifies lesions using three outcomes, based on the degree of 3-D morphological disorganization of the lesion. The first outcome describes "high disorganization" lesions (such as malignant melanoma, melanoma in situ, high-grade dysplastic nevi, and atypical melanocytic proliferation), which are candidates for biopsy. The second outcome is "non-evaluable", and biopsy decision for these is based on careful evaluation of other clinical criteria.

The MelaFind scanner is suitable for noninvasive assessment of lesions that are sufficiently pigmented to be clearly discerned from the surrounding normal skin using automated image-processing tools. Their diameter should be in the range between $2 \mathrm{~mm}$ and $22 \mathrm{~mm}$, and this dimension is determined by the imaging optics. Other restrictions and requirements involve accessibility by the handheld component, intact skin without scars, fibrosis, or foreign matter. MelaFind is not suitable for use on several anatomic sites, such as acral, palmar, plantar, mucosal, or subungual areas, as well as near the eyes.

The handheld component of the MelaFind imaging system consists of a radiation source (illuminator) that sequentially irradiates the lesion with 10 wavelengths of light (therefore, it is classified as a multispectral system that collects information in the spatial domain), including visible and near-IR bands. Light scattered back from regions beneath the surface of the lesion is collected by a lens system that focuses it onto a detector, forming one image for each of the wavelengths. The spatial resolution of the detector is around 3 melanocytes $(20 \mu \mathrm{m})$. Multispectral data are captured from a region up to $2.5 \mathrm{~mm}$ deep into the skin, and they provide unique 3 -D information regarding the morphological organization of the lesion. The information collected by the detector (ten images) is next analyzed by MelaFind's automatic data-analysis algorithms. The data-processing steps include calibration (reduction of noise and artifacts), followed by quality control (detection of imaging problems such as overexposure, underexposure, inappropriate lesion size, too much hair, etc). When problems are detected, the system instructs the operator to rescan the lesion.

The data-processing algorithms include the application of image-processing tools to the data received by the detector, such as lesion segmentation to identify portions of the image that belong to the lesion, followed by feature extraction to quantify the parameters that characterize lesions. Parameters of the lesion, such as asymmetry, color variation, and border changes (characteristics similar to the ABCDE criteria, evaluated by the computer) are quantified for the ten wavelengths used by MelaFind. There are differences in the skin's response (penetration depth, scattering characteristics) to the ten wavelengths used, and the differences between the images recorded at different wavelengths yield useful information for the decision-making process. The collected data are compared with information stored in the proprietary database of melanomas and benign lesions assembled by MelaSciences. The database was developed, trained, and tested with over 10,000 in vivo lesions and corresponding histological results in over 7000 patients. The sample included 600 melanomas, most of which were early stage lesions. This lesion database evolves and "learns" whenever a new case is evaluated and its parameters added to the database. The final element of the lesion assessment is classification based on the level of disorganization. MelaFind is currently in phase III clinical trials, and the technology holds the promise of being incorporated into clinical practice after further research and evaluation.

MelaFind is a type II instrument according to the classification introduced in this review, and early clinical studies are very promising. ${ }^{22}$ MelaFind also holds the promise of potentially becoming a screening device (type I in this 
review) after being proven as a type II device and appropriate modifications. Since the method requires irradiation by ten wavelengths, implementation as a type III scanner poses significant challenges, and it is less likely to prove feasible for full-body screening applications. Being recognized as a test method by insurance carriers would be a key step towards using diagnostic instruments to detect early melanoma, and extensive clinical validation will be needed to achieve this goal.

\section{Multispectral imaging in the frequency domain: spectroscopic methods}

Multispectral methods (in the spectral domain with radiation data for a point or region presented as intensity as a function of frequency or wavelength) are widely used in engineering for the characterization of materials, and they have also found their way into ex vivo and more recently into in vivo medical diagnostics. In this review advances and important features of IR and Raman spectroscopy as representatives of spectroscopic methods are summarized.

IR and Raman spectroscopy provide details regarding the chemical composition and molecular structure of substances in cells and biological tissues, and they are considered to be vibrational spectroscopic techniques..$^{23,24}$ Over time, with the progress of technology, they have also evolved into effective visualization tools. Since pathological changes and diseases cause chemical and structural changes in tissues on the molecular level, the resulting differences in the vibrational spectra serve as sensitive markers or unique fingerprints of the disease. ${ }^{25}$ These spectra can be captured by spectroscopic methods, which are noninvasive and do not require the administration of contrast. Early biomedical applications of these two spectroscopic methods would allow the measurement of spectra at a particular point in the tissue, and required therefore knowledge of the exact location of diseased tissue. With advances in optical imaging systems over the past 15 years, sensitive, high-throughput instruments have become available. These enhanced instruments collect a large number of spectra from larger samples faster and with improved spatial resolution with the aid of a microscopic imaging system. Also, over the past decade, fiber-optic probes have been developed, and their feasibility for in vivo applications has been demonstrated. Both spectra - the IR absorption spectrum and the Raman scattering spectrum - carry the same type of information regarding the energies of molecular vibrations. The underlying physics for these two spectroscopic methods is different, and each method offers specific advantages (and disadvantages) in medical applications.

\section{IR spectroscopy}

IR spectroscopy measures absorbed radiation, and can serve as a visualization tool to aid the pathologist in evaluating tissue specimens. IR instrumentation is usually less complex than devices for Raman spectroscopy that measure scattered light. Collection of IR data is faster, and signal-to-noise ratios are higher than for Raman spectrometers. The disadvantage of the method is that sample thickness for aqueous biological systems is limited to a few micrometers in transmission or attenuated total reflection (ATR) measurements. This limitation is imposed by water being a strong IR-absorbing medium. These samples are prepared on special substrates, and it is important to avoid affecting the composition of the sample during the preparation. Advanced Fourier transform IR (FTIR) spectrometers allow two-dimensional (2D) and 3D mapping of relevant spectral characteristics of the tissue. In IR spectroscopic imaging, the field of view is irradiated and imaged onto a detector array whose elements are sensitive to IR radiation. Each sensor element in the array will collect a spectrum, and the number of spectra collected simultaneously depends on the number of sensor elements in the array. IR radiation can be guided to the sample, and the reflected or transmitted radiation routed to the spectrometer, by means of IR fiber optics. Fiber-optic systems are suitable for in vivo endoscopic measurements; however, contact between the tissue and probe and probe contamination remain challenges to be resolved in the future.

\section{Raman spectroscopy}

Since the presence of water affects Raman spectra little, Raman spectroscopy is better suited for medical diagnostic applications. Raman spectra are typically measured in the reflection mode, and Raman spectroscopy can also be applied in vivo. Penetration of light can vary, depending on the wavelength, power, and sample properties (geometry and composition).

Human skin is a multilayered system, with layer thicknesses in the 10-500 $\mu \mathrm{m}$ range. Because of the absorption of IR radiation by water, the depth of penetration of IR radiation in skin tissue in in vivo IR spectroscopy measurements is only a few micrometers. Therefore, the ATR mode of spectroscopy can be used to analyze the outermost layer of stratum corneum. ${ }^{26}$ FTIR spectroscopy has also been used to analyze healthy skin and lesions. ${ }^{27-29}$ The ATR technique coupled to IR fiber optics has been applied for FTIR imaging of the stratum corneum to evaluate intact, thin skin sections, and the effects of exogenous materials..$^{30,31}$ FTIR spectroscopy has been combined with Raman imaging 
to analyze the stratum corneum and the permeation of lipids into the skin. ${ }^{32-34}$ Confocal Raman microspectroscopy has also been used to study structures in the stratum corneum ${ }^{35-37}$ in up to $200-\mu \mathrm{m}$ depth. The combination of Raman spectroscopy and Confocal Scanning Laser Microscopy (CSLM) offers the ability to analyze sections and layers of the skin without physically dissecting the tissue. ${ }^{38}$ The feasibility of these methods for the diagnosis of melanoma and other skin cancers has been demonstrated in several studies. ${ }^{39-43}$

Spectroscopic methods are very promising tools for the early diagnosis of melanoma. In addition to ex vivo characterization of diseased tissue, current research is focusing on in vivo applications. After adequate calibration with a large number of samples, automated evaluation of the data should be possible. Spectroscopic systems are best suited for type II devices, and a simplified instrument could possibly be converted into a type I device for screening purposes. Since the investigated tissue has to be irradiated with EM radiation of appropriate wavelength, it would be hard to accomplish both irradiation and the capturing of the scattered and reflected radiation for a large surface area in full-body measurements in type III devices.

\section{Terahertz imaging}

$\mathrm{THz}\left(1 \mathrm{THz}-10^{12} \mathrm{~Hz}\right)$ imaging uses radiation whose frequency lies between the microwave and IR regions (longer than IR wavelengths) of the EM spectrum. Until recently, it was the least explored and utilized region of the spectrum, due to difficulties involved in building $\mathrm{THz}$ radiation sources and detectors. Advances in optics and instrumentation over the past two decades have led to the development of a range of sources and detectors. One of the many applications of $\mathrm{THz}$ imaging is in the detection of disease, in particular skin cancer. The aim is to develop small, portable, fast, handheld scanners for early detection of cancer. The technology has shown promise; however, more research and development is needed to develop an imaging system capable of reliable automated detection of melanoma.

\section{Infrared imaging in the spatial domain: infrared thermography revisited}

The advances in IR (thermographic) imaging technology that captures spatial IR-emission data (rather than spectral characteristics), computers, and image-analysis techniques over the past two decades has led to renewed interest in IR imaging, in particular quantitative imaging, in a variety of medical and engineering applications. Since cancerous lesions are metabolically more active than healthy tissue and blood supply to the lesion is increased, cancerous lesions are warmer, and under certain conditions the difference in the IR radiation emitted by the lesion and healthy tissue can be readily detected by thermographic imaging.

Thermal imaging has been used as a research tool in medicine for over 50 years ${ }^{44-47}$ to analyze medical conditions associated with changes in the body or skin temperature. Skin cancer and melanoma detection are some of the earliest applications of IR imaging, and the value of the method has been a subject of controversy in the past. However, recent studies with advanced instrumentation and image-analysis tools have shown characteristic differences in thermal signatures between healthy skin or benign lesions and cancerous lesions. ${ }^{4-50}$ Some new results and methods will be discussed in this review. In this section, we highlight general issues and challenges in thermographic imaging as well as our recent efforts in quantitative thermal imaging of melanoma.

Thermographic techniques capture the EM radiation naturally emitted by the subject, the human body, and this radiation closely resembles the black-body radiation at $36^{\circ} \mathrm{C}-37^{\circ} \mathrm{C}$; its spectrum is shown in Figure 1 (top right). The emissivity of the skin is in the range 0.96-0.98. The capturing of naturally emitted radiation is an advantage over methods that require irradiation of the skin by an external source (to measure the reflected or scattered radiation in order to analyze skin structures) such as by MelaFind, in particular for full-body imaging applications. Therefore, the method is inherently safe and noninvasive. The emitted radiation is collected by the imaging optics and focused onto the focal plane array sensitive to IR wavelengths, thereby delivering the information in spatial (contrasted to spectral information in spectroscopic methods) and two dimensional (2-D) format. Recently, high-sensitivity uncooled detectors called focal plane arrays have become available, which have greatly simplified the technology, with the promise of significant cost reductions in the future. Another favorable feature of the method is that it is both image-forming (it allows the visual mapping of thermal structures on the skin surface and underneath the skin) and quantitative (allows measurement of temperature, metabolic activity rates, and other thermophysical properties based on temperature data). Depending on the number of detectors in the focal planearray matrix, a single image can contain 2D information with thousands of temperature measurements recorded in a fraction of a second. Modern IR cameras offer excellent time resolution, so the temperature changes of the subject can be recorded over time in the form of a movie. Images can be 
displayed as gray-scale or color coded with a color palette. The rainbow palette is preferred in medical applications, with the blue corresponding to low and the red to high temperature. Assigning absolute temperatures to emitted radiation poses a significant challenge and requires careful calibration of the instrument, as well as standardization of the imaging process. In addition to the visual information in the form of a 2D image or movie, quantitative information about the temperature evolution of a point or a region over time can be extracted from the 2-D information by postprocessing.

The value of thermographic imaging goes beyond providing visual insight into the thermal characteristics of the skin and the human body: proper processing of the temperature data offers the possibility of quantitative comparison and automated detection and quantification of abnormal events or processes or establishing other associations with medical conditions of interest. ${ }^{51-55} \mathrm{~A}$ good understanding of the thermal physiology of the body is essential for the interpretation of temperature data and spatiotemporal temperature changes in thermographic images. To enhance the understanding and interpretation of the information contained in thermographic images, computational modeling of the physiological processes has to go hand in hand with the thermographic imaging steps. ${ }^{52}$ When applying thermography in medical diagnostics, the voluntary and involuntary movement of the subject during the imaging process introduces an additional complexity into the image-analysis and quantification process. While specific image features are easy to detect and track in a series of images recorded over time in white-light imaging, the same features are not readily visible in the IR image sequence. Therefore, motion-tracking procedures developed specifically for IR imaging are needed for quantitative thermographic imaging.

IR imaging can be implemented either as a passive (static) or an active (dynamic) visualization method. Active IR imaging involves introducing external forcing, such as heating, cooling (also called thermostimulation in breast cancer imaging), or pressure to induce or enhance relevant thermal contrasts between the investigated lesion and healthy tissue. The general advantage of active measurement methods is that they do not require the subject to reach a well-defined steady state, which can be very time-consuming and challenging. For example, in the past, when static applications prevailed, the patient was required to spend one or more hours in a thermally conditioned exam room in order to fully acclimate (reach a thermal steady state) to the environment. Reaching a true steady state may not always be possible, and individual variations between test subjects will remain. Therefore, standardization in the imaging process - the key to comparison of images recorded at different times or of different subjects - was and remains a significant challenge in passive imaging.

In the dynamic method, the thermal response to an excitation, often applied as a step change in the thermal boundary conditions, involving the application or removal of heating or cooling, is measured. This is a much faster and more robust approach than the passive method. The dynamic method is well established in numerous engineering tests and applications, such as thermophysical property measurements, and it offers advantages in clinical applications, for which the duration of the measurement and the ease of use are critical. It allows the detection of abnormal features by self-referencing, ie, comparing the responses of healthy and diseased tissue of the same subject, rather than comparing responses of different subjects. While the application of external forcing increases the complexity of the measurement, the benefits outweigh the drawbacks. The required heating or cooling has to induce small changes in skin temperature; therefore, the duration of cooling is short and the temperature levels applied do not cause significant discomfort to the patient.

When the skin surface is cooled in active IR imaging, the difference in the thermophysical properties of the lesion (located near or underneath the surface) when compared to healthy tissue results in identifiable temperature contours and temperature differences between the lesion and the surrounding healthy tissue during the thermal recovery phase. These surface-temperature contours will differ from those present in the steady-state situation observed during passive IR imaging. Therefore, the measurement process consists of three phases: (1) the initial phase, when the skin is exposed to nominal ambient conditions, (2) the cooling phase, which is followed by (3) the thermal recovery phase.

In the Heat Transfer Lab of Johns Hopkins University, we developed a thermographic imaging system that allows accurate measurements of transient skin-surface temperature distributions ${ }^{51,53-57}$ and can be used for the detection of melanoma. It relies on active (dynamic) IR imaging based on cooling for the characterization of the thermal response of skin lesions and healthy skin. Cancerous lesions, such as melanomas, generate more heat and reheat faster than the surrounding healthy skin, thereby creating a marker for melanoma detection. The aim of the imaging is to distinguish benign lesions (which behave thermally similar to the healthy skin) from malignant pigmented lesions. 
The steps in the imaging process are illustrated in Figure 3. The imaging starts with applying a rectangular template to the region of interest, centered around the lesion. A whitelight photograph of the domain is taken first, followed by an IR image of the reference steady-state situation under ambient conditions (Figure 3A). The lesion is not visible in the reference steady-state IR image, implying that the heat generation is too small to be measured at the skin surface under static conditions. The skin is then cooled by blowing cold air or applying a cooling patch at $15^{\circ} \mathrm{C}-25^{\circ} \mathrm{C}$ for a duration of up to 1 minute. After removing the cooling load, the dynamic thermal response of the structure is acquired using IR imaging. Color-coded IR images recorded 2 seconds into the thermal recovery are shown in Figure 3B. The results suggest that thermal contrast between the lesion and the healthy surrounding tissue is enhanced by the cooling. After
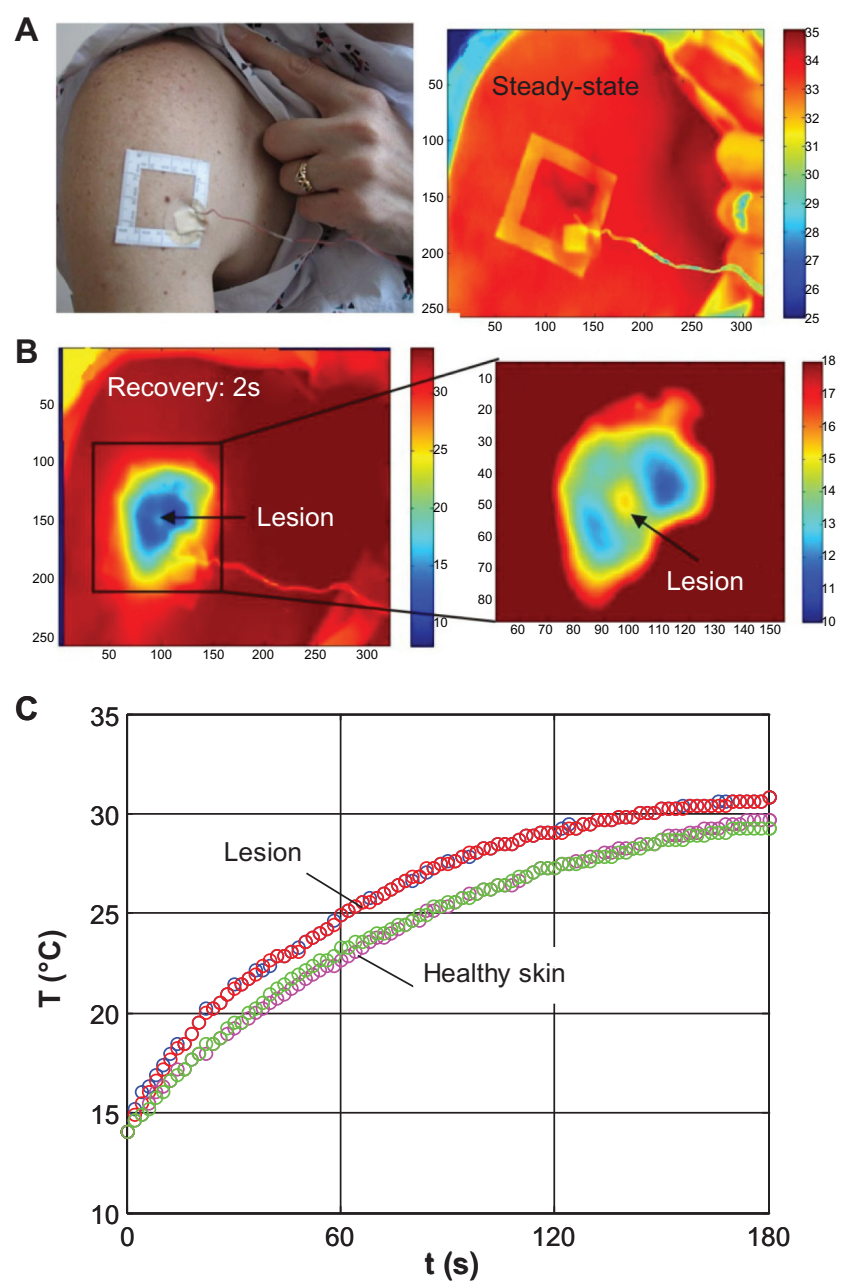

Figure 3 (A) White light photograph of the larger body surface area with a cluster of pigmented lesions, adhesive window serving as thermal marker and reference IR image of the region at ambient temperature; (B) the same area $2 \mathrm{~s}$ into the thermal recovery and magnified section of the melanoma lesion and its surroundings; (C) temperature profiles of the lesion and the surrounding normal skin during the thermal recovery process. ${ }^{51-53}$ the postprocessing steps, the temperature of the lesion and healthy tissue are plotted as a function of time during the thermal recovery, as shown in Figure 3C. There is a significant difference in temperature between the melanoma lesion and healthy tissue during thermal recovery, whereas benign pigmented lesions have the same thermal recovery as the healthy tissue. The temperature difference can be measured accurately using modern IR cameras. More details about the method are available in the literature. ${ }^{51-57}$ This imaging technique holds the promise of staging melanomas based on the magnitude of temperature differences and other thermal characteristics of the lesion during the recovery process.

\section{Motion tracking in dynamic IR imaging}

IR imaging is a noncontact method, and this feature offers advantages in terms of ease of application and the ability to image larger surface areas and multiple lesions. Therefore, this method is suitable for type III full-body imaging systems. However, during the thermal recovery phase, involuntary movement of the patient is unavoidable in a clinical environment, and even breathing can cause small spatial displacement of lesions that can lead to deterioration of the measurement data. The measurement involves accurate tracing of the transient temperature response at any specific point on the skin. To accomplish this, it is necessary to apply motion tracking/compensation processing on the IR video sequence. The framework of motion tracking can be generally summarized in two steps. ${ }^{57}$

\section{Step I: registration between the white- light image and the first IR image frame}

Since the lesion is visible in the white-light image only, to identify its corresponding points in the IR image, the first task is the registration via feature point correspondence. An adhesive marker, visible both in white-light and IR images, is used for the registration computations. As shown in Figure 4A, the four corners of the marker are first identified in the white-light image either manually or by an edge-detection algorithm, such as the Harris method. ${ }^{58}$ Next, the corresponding points are identified in the first IR image frame, which serves as the reference image in step 2 (Figure 4B). Based on the four pairs of points for coordinate correspondence, a 2-D projective transformation matrix can be identified to map any point in the white-light image to its corresponding point in the reference IR image frame. ${ }^{59}$ The lesion region can be delineated in the white-light image via an interactive segmentation algorithm, such as a 

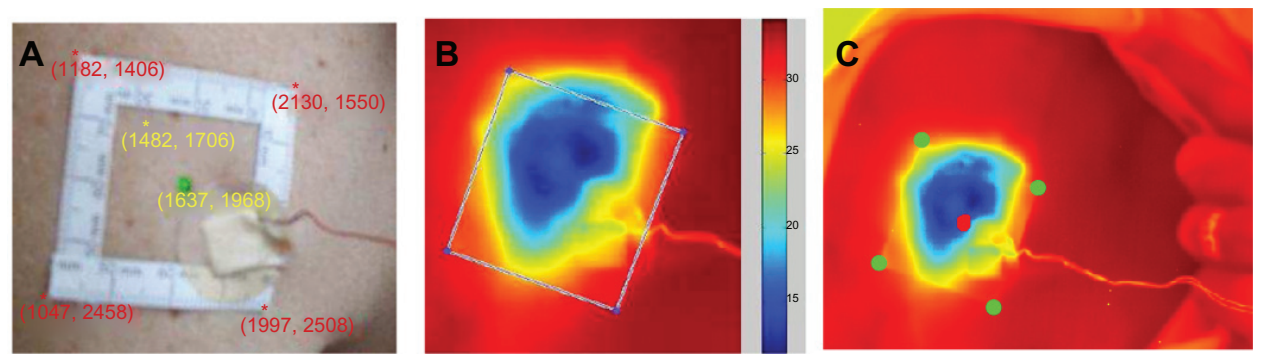

Figure 4 Image registration steps in melanoma detection: (A) white light image with characteristic points for image analysis (rectangular marker and lesion center), (B) edge and corner correspondence in IR image, (C) lesion contour registration in the IR images via transformation matrix.

random walker. ${ }^{60}$ Once the transformation matrix is solved, the delineated lesion can be transferred to the registered IR image (Figure 4C).

\section{Step 2: registration between the consecutive IR image frames in the recovery IR video sequence}

After the lesion location is identified in the first IR image frame, registration can be applied between every consecutive IR frame in the video sequence. Any point of interest can then be tracked in the video in the presence of involuntary subject movement. The registration between the consecutive IR frames can be achieved using a quadratic motion model. ${ }^{61}$

Transformations of the image during the motioncorrection steps are illustrated in Figure 5. The magnitude and direction of the motion are indicated as a vector in each image frame. A sequence of IR image frames recorded during the recovery phase can be aligned to compensate for involuntary body/limb movement of the patient by applying a quadratic motion model for registration. The registration of the lesion region enables an accurate comparison between

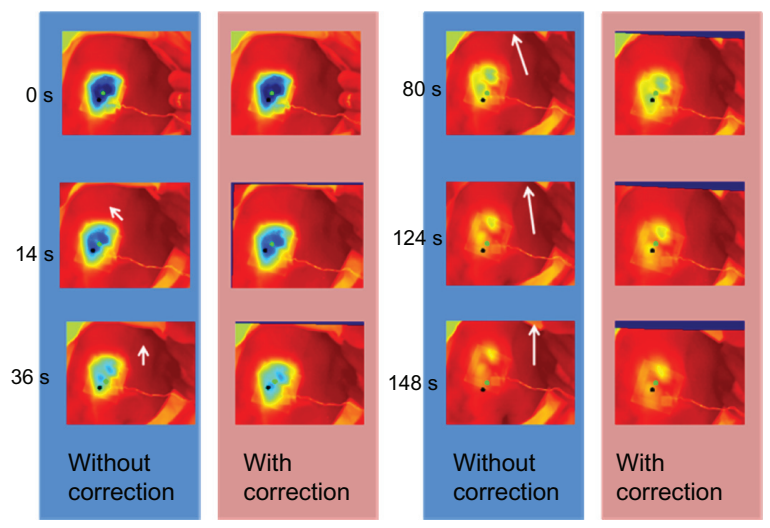

Figure 5 Motion correction for compensation of the subject's involuntary movement in IR images recorded at different time instants.

Notes: The effects of motion correction can be observed in the corrected columns as dark regions around the edges of the images. White arrows in the uncorrected images represent the direction and magnitude of motion of the subject. the transient thermal response of healthy skin and that of the lesion, and provides critical information that allows identification of the malignant lesion, as shown by Pirtini Cetingul and Herman. ${ }^{51,52,54}$ Since IR imaging (with proper calibration) yields quantitative data, the method (analysis of the thermal recovery process) holds the potential of allowing the staging of the disease.

This version of quantitative thermography has the potential to be implemented as a type I, type II, and type III imaging device, since the radiation naturally emitted by the body is measured, and direct contact between the skin and the probe is not required. The drawback is the need for sophisticated motion tracking in the image-processing phase to compensate for involuntary movement of the subject. IR thermography alone cannot distinguish between different types of skin cancer; however, it can alert the patient to the need to seek out professional help (type I device for screening). As a type II device, with proper calibration, it could potentially measure the magnitude of excess heating (caused by increased metabolic activity and blood supply in the cancerous lesion) and provide a quantitative measure of the malignant potential. The discipline of IR thermography has been experiencing dramatic growth over the past decade, primarily driven by night vision and surveillance applications, accompanied by improvements in hardware and reduction in cost of the instrumentation. With current trends continuing, thermography would be a good option for large-scale screening efforts at the level of the patient or in primary care facilities.

\section{Confocal scanning laser microscopy}

CSLM is a noninvasive imaging method that has the ability to assess the cellular and nuclear details of skin lesions in vivo, with details similar to histology. ${ }^{62,63}$ CSLM detects backscattered light, and contrast is caused by natural variations of the refractive index of tissue microstructures,${ }^{64}$ such as organelles and melanosomes. Since the skin pigment melanin has a high 

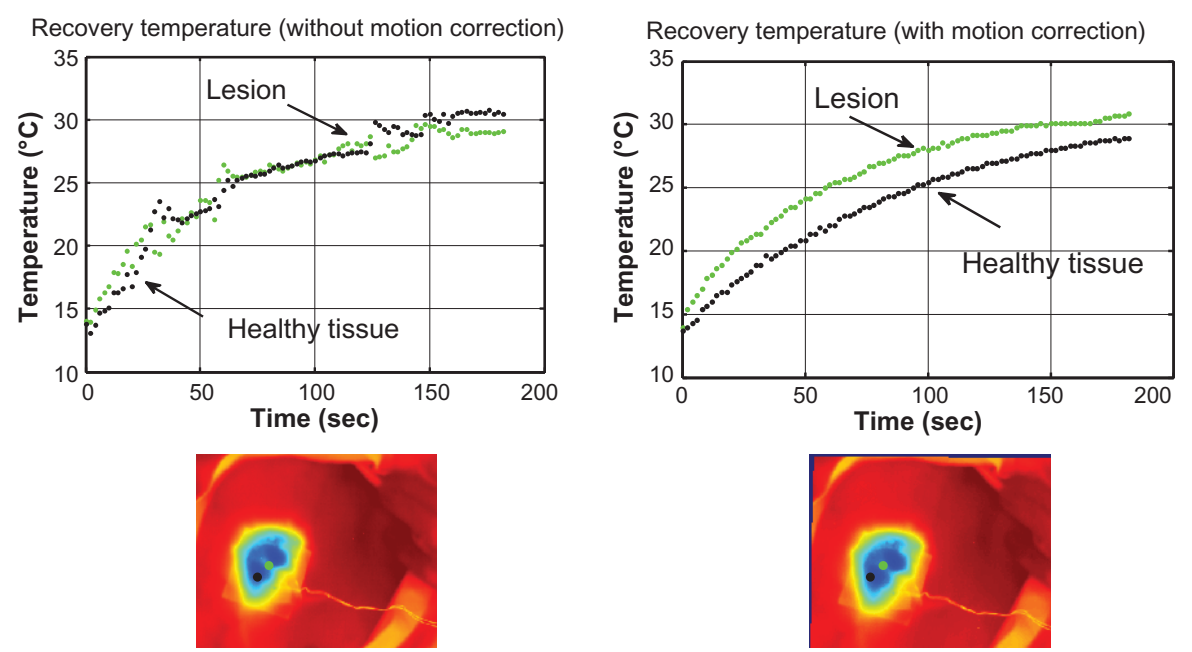

Figure 6 Impact of the motion correction on the reconstructed temperature profiles.

Notes: Without motion correction (left), the noise is of the order of magnitude of the measured signal. With motion correction. the temperature of the melanoma lesion is higher than the temperature of the surrounding healthy tissue. This temperature difference can be used to quantify the malignant potential of the cancerous lesion.

refractive index, it behaves as a contrast agent for cytoplasm. ${ }^{65}$ Therefore, the cytoplasm of melanocytes in pigmented and amelanotic melanomas appears brighter and can be easily detected. Past small-scale clinical studies focused on the detection of skin cancers, such as melanomas, ${ }^{66-69}$ basal and squamous cell carcinomas, ${ }^{70}$ benign lesions, and the detection of margins between lesions and surrounding normal tissue. ${ }^{71}$ Clinical studies have shown that the technique has a sensitivity in the range of $89 \%-97 \%$ and a specificity in the range of $80 \%-85 \%$ (depending on the study), ${ }^{62-71}$ after appropriate training of the physician in the interpretation of the patterns observed using CSLM. Similar to other diagnostic methods that involve interpretation of visual images, the diagnostic accuracy will be dependent on the experience of the operator.

A commercially available CSLM imaging system (VivaScope) is shown in Figure 7A. The method is capable of assessing parallel layers of the skin up to a depth of $300 \mu \mathrm{m}$ (Figure 7B). The head of the imaging system contains the optical components (light source and imaging optics) as well as a computer-controlled linear translation stage for sequential scanning of the area of interest. To minimize
A

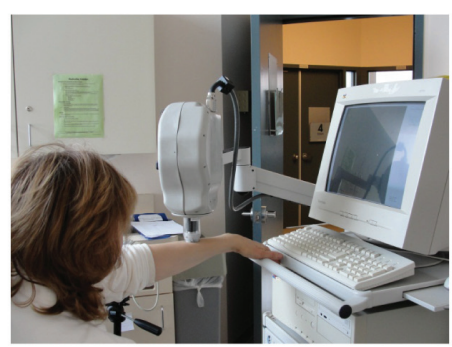

B

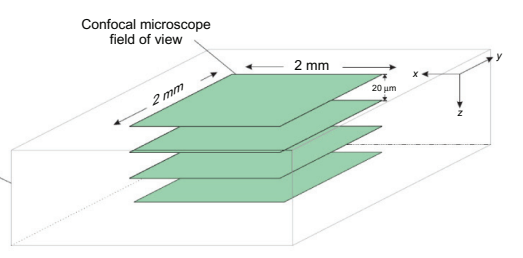

C
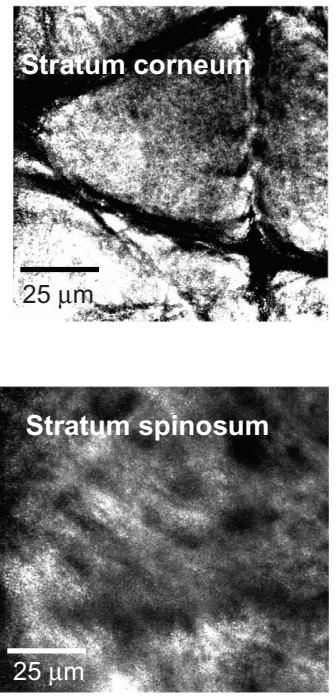
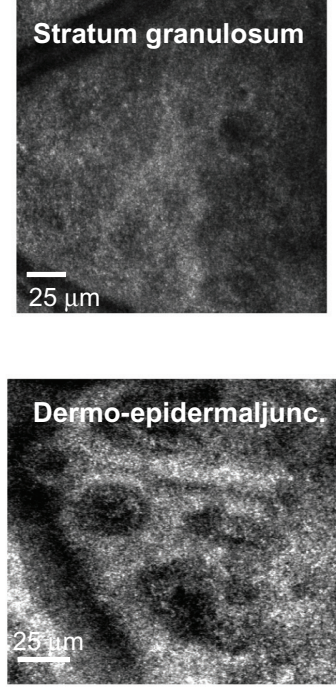

Without a mole

Figure 7 (A) VivaScope imaging system for dermatology; (B) parallel skin layers imaged by the CSLM system; (C) CSLM images of different skin layers. 
optical losses between media with very different refractive indices, an immersion fluid is applied between the skin and the optics to enable direct contact between detector and skin. The positioning of the detector head relative to the lesion may pose a challenge for some lesion locations. A CSLM system and images of four skin layers are displayed in Figure 7, and they deliver fine detail and high-quality information on skin structures.

CSLM is a commercially available type II imaging system suitable for use in specialized centers by trained operators. Since it requires direct contact between optics and the skin by means of an immersion fluid, this imaging system is not suitable for a full-body scanner. Also, since the interpretation of the images requires specialized knowledge and automated interpretation of the images would pose a major challenge, it is not likely that CSLM will be suitable as a type I screening instrument.

\section{Optical coherence tomography}

OCT is a noninvasive, in vivo imaging method, which captures high-resolution $(\mu \mathrm{m}), 3 \mathrm{D}$ images of biological tissue. OCT is an interferometric technique using relatively long-wavelength light in the near-IR portion of the spectrum, which is able to penetrate into the scattering medium (deeper than CSLM). This technique has higher resolution, greater detection depth and image size, and faster scanning time than CSLM. Although in OCT, a melanoma shows increased light scattering and more homogenous signal distribution than healthy skin, more work is needed to improve its utility for skin cancer detection. ${ }^{12,13} \mathrm{OCT}$ is suitable as a type II device for use in specialized centers by operators trained in the interpretation of the images.

\section{Conclusions}

This brief review discusses some of the promising current technologies as well as the needs and challenges in developing sensitive and reliable diagnostic tools for the early detection of melanoma. Melanoma is the fastestgrowing cancer in terms of incidence, and the need for accurate diagnostic tools is increasing. Every year, around 2.5-3 million skin lesions are evaluated in the US, and over 100,000 are diagnosed as melanoma. The objective is to develop automated diagnostic instruments for screening of individual lesions and full-body screening, as well as sophisticated instruments that can provide dermatologists with fine detail regarding the structure of a lesion and staging information in vivo. Screening instruments would alert patients to seek the care of a dermatologist, and would be intended for use in primary care facilities or by the patients, similar to blood pressure cuffs or diabetestesting equipment. With recent progress in electronics and instrumentation, several sophisticated and very promising imaging methods have emerged and are being investigated in small trials. One of the key challenges is that diagnostic instruments are expected to compete in price and ease of use with visual inspection, which is the current standard of care. Clearly, using a diagnostic instrument would increase the duration and the cost of the exam; therefore, insurance coverage would be a key driving factor for technology development. The funding for technology development beyond the initial feasibility studies and the funding of large scale studies to demonstrate the effectiveness of imaging systems along with the complex and lengthy governmental approval process are the main challenges on the path of these imaging systems finding their place in medical care.

\section{Acknowledgments}

Images and photographs for this review were taken or contributed by Rajeev Hatwar, Tze-Yuan Cheng, and Muge Pirtini Cetingul. This research was funded by the National Institutes of Health NCI (grant no 5R01CA161265-02), the National Science Foundation (grant no 0651981), and the Alexander and Margaret Stewart Trust through the Cancer Center of Johns Hopkins University.

\section{Disclosure}

The author reports no conflicts of interest in this work.

\section{References}

1. National Cancer Institute. SEER Stat Fact Sheets: Melanoma of the Skin. 2012. Available from: http://seer.cancer.gov/statfacts/html/melan.html. Accessed August 3, 2012.

2. Skin Cancer Foundation. Skin Cancer Facts. 2012. Available from: http:// www.skincancer.org/Skin-Cancer-Facts. Accessed August 3, 2012.

3. Jemal A, Siegel R, Ward E, Hao Y, Xu J, Thun MJ. Cancer statistics, 2009. CA Cancer J Clin. 2009;59(4):225-249.

4. Elder D. Tumor progression, early diagnosis and prognosis of melanoma. Acta Oncol. 1999;38(5):535-547.

5. Fecher LA, Cummings SD, Keefe MJ, Alani RM. Toward a molecular classification of melanoma. J Clin Oncol. 2007;25(12):1606-1620.

6. Wartman D, Weinstock M. Are we overemphasizing sun avoidance in protection from melanoma? Cancer Epidemiol Biomarkers Prev. 2008; 17(3):469-470.

7. Geller AC, Swetter SM, Brooks K, Demierre MF, Yaroch AL. Screening, early detection, and trends for melanoma: current status (2000-2006) and future directions. J Am Acad Dermatol. 2007;57(4):555-572; quiz 573-576.

8. Friedman RJ, Rigel DS, Kopf AW. Early detection of malignant melanoma: the role of physician examination and self-examination of the skin. CA Cancer J Clin. 1985;35(3):130-151.

9. Abbasi NR, Shaw HM, Rigel DS, et al. Early diagnosis of cutaneous melanoma: revisiting the ABCD criteria. JAMA. 2004;292(22): $2771-2776$. 
10. Thomas L, Tranchand P, Berard F, Secchi T, Colin C, Moulin G. Semiological value of $\mathrm{ABCDE}$ criteria in the diagnosis of cutaneous pigmented tumors. Dermatology. 1998;197(1):11-17.

11. Wang SQ, Rabinovitz H, Kopf AW, Oliviero M. Current technologies for the in vivo diagnosis of cutaneous melanomas. Clin Dermatol. 2004;22(3):217-222.

12. Psaty EL, Halpern AC. Current and emerging technologies in melanoma diagnosis: the state of the art. Clin Dermatol. 2009;27(1):35-45.

13. Patel JK, Konda S, Perez OA, Amini S, Elgart G, Berman B. Newer technologies/techniques and tools in the diagnosis of melanoma. Eur J Dermatol. 2008;18(6):617-631.

14. Andreassi M, Andreassi L. Utility and limits of noninvasive methods in dermatology. Expert Rev Dermatol. 2007;2(3):249-255.

15. http://www.melafind.com.

16. http://www.melasciences.com.

17. Gutkowicz-Krusin D, Elbaum M, Jacobs A, et al. Precision of automatic measurements of pigmented skin lesion parameters with a MelaFind(TM) multispectral digital dermoscope. Melanoma Res. 2000;10(6):563-570.

18. Elbaum M, Kopf AW, Rabinovitz HS, et al. Automatic differentiation of melanoma from melanocytic nevi with multispectral digital dermoscopy: a feasibility study. J Am Acad Dermatol. 2001;44(2):207-218.

19. Elbaum M. Computer-aided melanoma diagnosis. Dermatol Clin. 2002;20(4):735-747, x-xi.

20. Elbaum M. Automated diagnosis: illustrated by the MelaFind system. In: Marghoob AA, Kopf AW, Braun R, editors. An Atlas of Dermoscopy. Abingdon: Taylor and Francis; 2004:325-341.

21. Friedman RJ, Gutkowicz-Krusin D, Farber MJ, et al. The diagnostic performance of expert dermoscopists vs a computer-vision system on small-diameter melanomas. Arch Dermatol. 2008;144(4):476-482.

22. Monheit G, Cognetta AB, Ferris L, et al. The performance of MelaFind: a prospective multicenter study. Arch Dermatol. 2011;147(2):188-194.

23. Rigel DS, Roy M, Yoo J, Cockerell CJ, Robinson JK, White R. Impact of guidance from a computer-aided multispectral digital skin lesion analysis device on decision to biopsy lesions clinically suggestive of melanoma. Arch Dermatol. 2012;148(4):541-543.

24. Krafft C, Sergo V. Biomedical applications of Raman and infrared spectroscopy to diagnose tissues. Spectroscopy. 2006;20(5-6) 195-218.

25. Garidel P. Insights in the biochemical composition of skin as investigated by micro infrared spectroscopic imaging. Phys Chem Chem Phys. 2003;5:2673-2679.

26. Lucassen GW, Caspers PJ, Puppels GJ. In vivo infrared and Raman spectroscopy of stratum corneum. Proc SPIE. 1998;3257:52-60.

27. Mendelsohn R, Flach CR, Moore DJ. Determination of molecular conformation and permeation in skin via IR spectroscopy, microscopy and imaging. Biochim Biophys Acta. 2006;1758(7):923-933.

28. Mendelsohn R, Rerek ME, Moore DJ. Infrared spectroscopy and microscopic imaging of stratum corneum models and skin. Phys Chem Chem Phys. 2000;2:4651-4657.

29. Mendelsohn R, Chen HC, Rerek ME, Moore DJ. Infrared microscopic imaging maps the spatial distribution of exogenous molecules in skin. J Biomed Opt. 2003;8(2):185-190.

30. Xiao C, Moore DJ, Flach CR, Mendelsohn R. Permeation of dimyristoylphosphatidylcholine into skin - structural and spatial information from IR and Raman microscopic imaging. Vib Spectrosc. 2005;38(1-2): $151-158$

31. Xiao C, Moore DJ, Rerek ME, Flach CR, Mendelsohn R. Feasibility of tracking phospholipid permeation from IR and Raman microscopic imaging. J Invest Dermatol. 2005;124(3):622-632.

32. Zhang G, Moore DJ, Mendelsohn R, Flach CR. Vibrational microspectroscopy and imaging of molecular composition and structure during human corneocyte maturation. J Invest Dermatol. 2006;126(5):1088-1094

33. Bommannan D, Potts RO, Guy RH. Examination of stratum corneum barrier function in vivo by infrared spectroscopy. J Invest Dermatol. 1990;95(4):403-408.
34. Bhargava R, Levin IW. Gram-Schmidt orthogonalization for rapid reconstruction of Fourier transform infrared spectroscopic imaging data. Appl Spectrosc. 2004;58(8):995-1000.

35. Xiao C, Flach CR, Marcott C, Mendelsohn R. Uncertainties in depth determination and comparison of multivariate with univariate analysis in confocal Raman studies of a laminated polymer and skin. Appl Spectrosc. 2004;58(4):382-389.

36. Caspers PJ, Lucassen GW, Carter EA, Bruining HA, Puppels GJ. In vivo confocal Raman microspectroscopy of the skin: noninvasive determination of molecular concentration profiles. J Invest Dermatol. 2001;116(3):434-442.

37. Caspers PJ, Lucassen GW, Puppels GJ. Combined in vivo confocal Raman spectroscopy and confocal microscopy of human skin. Biophys J. 2003;85(1):572-580.

38. Percot A, Lafleur M. Direct observation of domains in model stratum corneum lipid mixtures by Raman microspectroscopy. Biophys $J$. 2001;81(4):2144-2153.

39. Tfalyli A, Piot O, Durlach A, Bernard P, Manfait M. Discriminating nevus and melanoma on paraffin-embedded skin biopsies using FTIR microspectroscopy. Biochim Biophys Acta. 2005;1724(3):262-269.

40. Lasch P, Naumann D. FT-IR microspectroscopic imaging of human carcinoma thin sections based on pattern recognition techniques. Cell Mol Biol (Noisy-le-grand). 1998;44(1):189-202.

41. Gniadecka M, Philipsen PA, Sigurdsson S, et al. Melanoma diagnosis by Raman spectroscopy and neural networks: structure alteration in proteins and lipids in intact cancer tissue. J Invest Dermatol. 2004;122(2):443-449

42. Nijssen A, Bakker Schut TC, Heule F, et al. Discriminating basal cell carcinoma from its surrounding tissue by Raman spectroscopy. J Invest Dermatol. 2002;119(1):64-69.

43. Huang Z, Lui H, Chen XK, Alajlan A, McLean DI, Zeng H. Raman spectroscopy of in vivo cutaneous melanin. J Biomed Opt. 2004;9(6): 1198-1205.

44. Cristofolini M, Piscioli F, Valdagni C, Della Selva A. Correlations between thermography and morphology of primary cutaneous malignant melanomas. Acta Thermogr. 1976;1(1):3-11.

45. Hartmann M, Kunze J, Friedel S. Telethermography in the diagnostic and management of malignant melanomas. J Dermatol Surg Oncol. 1981;7(3):213-218.

46. Di Carlo A. Thermography and the possibilities for its applications in clinical and experimental dermatology. Clin Dermatol. 1995;13(4): 329-336.

47. Diakides NA. 1998 Infrared imaging: an emerging technology in medicine. IEEE Eng Med Biol Mag. 1998;17(4):17-18.

48. Fauci M, Breiter R, Cabanski W, et al. Medical infrared imaging differentiating facts from fiction, and the impact of high precision quantum well infrared photodetector camera systems, and other factors, in its reemergence. Infrared Phys. 2001;42(3-5):337-344.

49. Gulyaev YV, Markov AG, Koreneva LG, Zakharov PV. Dynamical infrared thermography in humans. IEEE Eng Med Biol Mag. 1995;14: 766-771.

50. Ring EFJ, Ammer K. Infrared thermal imaging in medicine. Physiol Meas. 2012;33(3):R33-R46.

51. Herman C, Pirtini Cetingul M. Quantitative visualization and detection of skin cancer using dynamic thermal imaging. J Vis Exp. 2011;(51): e2679.

52. Pirtini Cetingul M, Herman C. Heat transfer model of skin tissue for the detection of lesions: sensitivity analysis. Phys Med Biol. 2010;55(19): 5933-5951.

53. Pirtini Cetingul M, Herman C. Quantification of the thermal signature of a melanoma lesion. Int J Therm Sci. 2011;50(4):421-431.

54. Pirtini Cetingul M, Herman C. The assessment of melanoma risk using the dynamic infrared imaging technique. J Therm Sci Eng Appl. 2011;3(3):031006.1-031006.9.

55. Pirtini Cetingul M, Alani RM, Herman C. Quantitative evaluation of skin lesions using transient thermal imaging. Proceedings of the International Heat Transfer Conference, IHTC14; August 8-13, 2010; Washington, DC, USA. 
56. Pirtini Cetingul M, Alani RM, Herman C. Detection of skin cancer using skin transient thermal imaging. Proceedings of the ASME 2010 Summer Bioengineering Conference, SBC2010; June 15-19, 2010; Naples, Florida, USA.

57. Pirtini Cetingul M, Cetingul HE, Herman C. Analysis of transient thermal images to distinguish melanoma from dysplastic nevi. Proceedings of the SPIE Medical Imaging Conference; February 12-17, 2011; Lake Buena Vista, FL, USA.

58. Harris C, Stephens M. A combined corner and edge detector. Proceedings of the 4th Alvey Vision Conference; 1988.

59. Hartley R, Zisserman A. Multiple View Geometry in Computer Vision. Cambridge: Cambridge University Press; 2003:32-33.

60. Grady L. Random walks for image segmentation, pattern analysis and machine intelligence. IEEE Trans Pattern Anal Mach Intell. 2006; 28(11): 1768 .

61. Odobez JM, Bouthemy P. Robust multiresolution estimation of parametric motion models. J Vis Commun Image Represent. 1995; 6(4):348-365.

62. Pawley JB. Handbook of Biological Confocal Microscopy. 2nd ed. New York: Plenum Press; 1995.

63. Webb RH. Confocal optical microscopy. Rep Prog Phys. 1996;59(3): $427-471$.

64. Rajadhyaksha M, Zavislan JM. Confocal reflectance microscopy of unstained tissue in vivo. Retin Lipid-Soluble Vitam Clin Pract. 1998;14(1):26-30.

65. Rajadhyaksha M, Grossman M, Esterowitz D, Webb RH, Anderson RR. In vivo confocal scanning laser microscopy of human skin: melanin provides a good contrast. J Invest Dermatol. 1995;104(6):946-952.
66. Busam KJ, Hester K, Charles C, et al. Detection of clinically amelanotic malignant melanoma and assessment of its margins by in vivo confocal scanning laser microscopy. Arch Dermatol. 2001;137(7):923-929.

67. Charles CA, Marghoob AA, Busam KJ, Clark-Loeser L, Halpern AC. Melanoma or pigmented basal cell carcinoma: a clinical-pathologic correlation with dermoscopy, in vivo confocal scanning laser microscopy, and routine histology. Skin Res Technol. 2002;8(4):282-287.

68. Marghoob AA, Charles C, Busam KJ, et al. In vivo confocal scanning laser microscopy of a series of congenital melanocytic nevi suggestive of having developed malignant melanoma. Arch Dermatol. 2005; 141(11):1401-1412.

69. Scope A, Benvenuto-Andrae C, Agero AL, et al. In vivo reflectance confocal microscopy imaging of melanocytic skin lesions: Consensus terminology glossary and illustrative images. $\mathrm{J} \mathrm{Am} \mathrm{Acad} \mathrm{Dermatol.}$ 2007;57(4):644-658

70. Aghassi D, Anderson RR, Gonzales S. Confocal laser microscopic imaging of actinic keratosis in vivo: a preliminary report. $J$ Am Acad Dermatol. 2000;43(1):42-48.

71. Busam KJ, Hester K, Charles C, et al. Detection of clinically amelanotic malignant melanoma and assessment of its margins by in vivo confocal scanning laser microscopy. Arch Dermatol. 2001;137(7):923-929.
Clinical, Cosmetic and Investigational Dermatology

\section{Publish your work in this journal}

Clinical, Cosmetic and Investigational Dermatology is an international, peer-reviewed, open access, online journal that focuses on the latest clinical and experimental research in all aspects of skin disease and cosmetic interventions. All areas of dermatology will be covered; contributions will be welcomed from all clinicians and

\section{Dovepress}

basic science researchers globally. This journal is indexed on CAS The manuscript management system is completely online and includes a very quick and fair peer-review system, which is all easy to use. Visit http://www.dovepress.com/testimonials.php to read real quotes from published authors. 